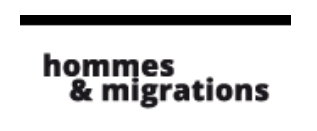

Hommes \& migrations

Revue française de référence sur les dynamiques

migratoires

$1282 \mid 2009$

Santé et droits des étrangers : réalités et enjeux

\title{
Hommes et Médiations. À propos du droit au séjour des étrangers malades
}

\section{Marie Hénocq et Arnaud Veïsse}

\section{Q OpenEdition \\ Journals}

\section{Édition électronique}

URL : http://journals.openedition.org/hommesmigrations/440

DOI : 10.4000/hommesmigrations. 440

ISSN : 2262-3353

\section{Éditeur}

Musée national de l'histoire de l'immigration

\section{Édition imprimée}

Date de publication : 1 novembre 2009

Pagination : 44-53

ISSN : 1142-852X

\section{Référence électronique}

Marie Hénocq et Arnaud Veïsse, "Hommes et Médiations. À propos du droit au séjour des étrangers malades », Hommes \& migrations [En ligne], 1282 | 2009, mis en ligne le 29 mai 2013, consulté le 19 avril 2019. URL : http://journals.openedition.org/hommesmigrations/440 ; DOI : 10.4000/ hommesmigrations. 440 


\section{Hommes et Médiations À propos du droit au séjour des étrangers malades}

Par Marie Hénocq, chargée de projet santé à la Cimade Île-de-France et Arnaud Veïsse, directeur du Comede

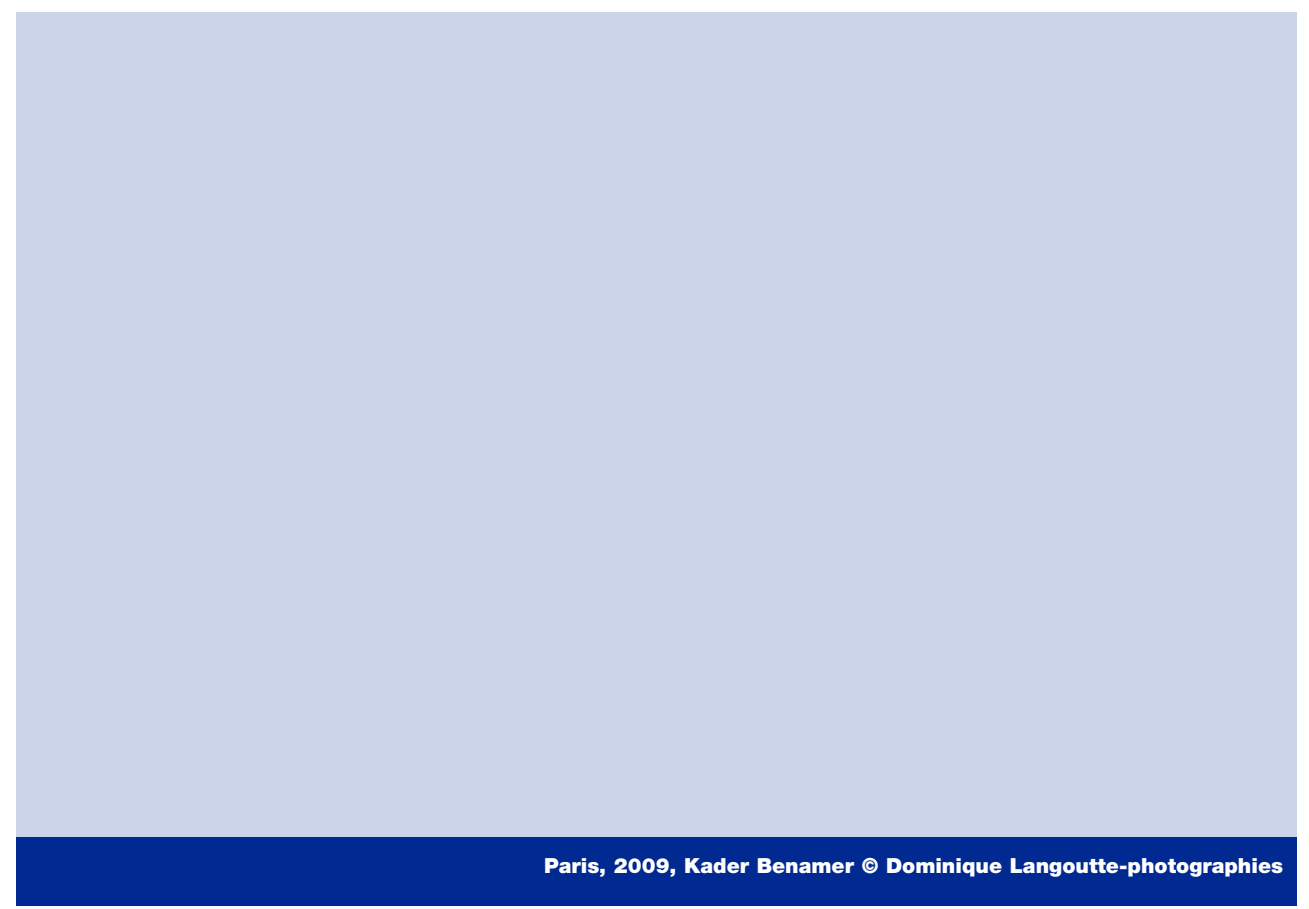

En France, le droit au séjour pour raison médicale existe depuis 1998. Les médecins représentent un maillon essentiel du processus d'application de ce droit. Pourtant, depuis 2002, les décisions préfectorales tiennent de moins en moins compte de leurs avis. Cette évolution restrictive de la procédure "étrangers malades" étonne et inquiète. Pour y voir plus clair, des associations comme la Cimade et le Comede tentent de jeter des ponts entre les intervenants du monde médical, administratif et judiciaire. Le point sur ces médiations. 
La mise en place depuis quelques années d'une politique de restriction d'application du droit au séjour des étrangers malades en France a rendu plus que jamais nécessaire l'interdisciplinarité des acteurs de ce dispositif, à savoir médecins, juristes, associatifs et administrations, et ce dans le respect du rôle de chacun. Comment appréhender l'état de santé des migrants qui sollicitent les permanences associatives quand on n'est pas médecin ? Comment se repérer dans les méandres des procédures de demandes de titre de séjour, de renouvellement ou encore de recours devant le juge administratif, voire de reconduite à la frontière, quand on est soignant? Peut-on appréhender ces situations en complémentarité avec des approches aussi différentes que le droit des étrangers et la pratique médicale?

C'est autour de ces questions qu'un partenariat original s'est constitué entre la Cimade et le Comede. Par son action de promotion de l'accueil, de la santé et de l'insertion des exilés en France, l'équipe du Comede est confrontée depuis trente ans aux tensions entre les politiques de santé publique et celles du contrôle de l'immigration. Face à une demande croissante de nature juridico-médicale de la part des étrangers soutenus dans ses permanences "migrants", ainsi que dans les centres de rétention administrative où le motif médical constitue parfois un obstacle à l'exécution imminente d'une mesure d'éloignement du territoire, la Cimade a fait appel au Comede en 2004. Il s'agissait de développer en commun des actions d'information et de formation destinées autant aux acteurs juridiques que médicaux, dans un objectif associé de respect du droit et de protection de la santé des étrangers. Poursuivi depuis 2007 dans le cadre de l'Espace Santé Droit basé à Aubervilliers, ce partenariat a démontré l'enjeu déterminant que constitue la médiation interprofessionnelle dans le domaine du droit à la santé des étrangers.

\section{Un démantèlement politique lourd de conséquences sur le terrain}

Adopté par l'Assemblée nationale en 1998, le droit au séjour pour raison médicale est reconnu pour l'étranger "résidant habituellement en France dont l'état de santé nécessite une prise en charge médicale dont le défaut pourrait entraîner pour lui des conséquences d'une exceptionnelle gravité, sous réserve qu'il ne puisse effectivement bénéficier d'un traitement approprié dans le pays dont il est originaire (1)". Par rapport aux autres procédures de régularisation pour motifs de "vie privée et familiale", la procédure "étrangers malades" présente la singularité de faire intervenir des médecins dans le processus de décision préfectorale. Face au malade étranger sollicitant la délivrance ou le renouvellement d'un titre de séjour, le médecin doit évaluer à la fois le risque 
d'exceptionnelle gravité du défaut de prise en charge médicale et le risque d'exclusion des soins nécessaires au pays d'origine. Mais avec la montée en charge du dispositif, cette expertise médico-juridique originale n'a pas tardé à révéler sa complexité(2). Au cours des premières années d'application, ce droit profite à un nombre croissant d'étrangers malades. L'augmentation régulière de la délivrance des premières cartes de séjour se poursuit jusqu'en 2004, avec un maximum observé de 7813 nouveaux étrangers malades, soit un nombre comparable à celui des demandeurs d'asile reconnus réfugiés sur la période. Mais à la différence des réfugiés, les étrangers malades doivent faire renouveler leur carte de séjour une ou plusieurs fois par an, ce qui accroît très vite la charge de travail des médecins et des préfectures. Ainsi, en 2005, pour 7612 étrangers malades nouvellement régularisés, les Médecins inspecteurs de santé publique (MISP) ont dû délivrer 40940 avis pour un total de 23614 demandeurs. En l'absence de moyens alloués aux administrations concernées, cette évolution est propice à la multiplication des obstacles à la procédure.

"Le droit au séjour des étrangers malades constitue la faille majeure du système" : dès 2002, le rapport Escoffier de l'Inspection générale de l'administration sur le réexamen des dossiers des étrangers en situation irrégulière annonce une période de restriction continue d'un droit qui, s'il reste inscrit dans la loi, reste géré dans une grande opacité. C'est pourquoi, avec l'apparition en 2003 d'une vague de refus de délivrance de cartes de séjour pour raison médicale, le Comede a mis en place un programme d'observation des réponses administratives et contentieuses aux demandes de protection faites par les étrangers malades. L'objectif est double: informer les malades et les professionnels concernés sur les pratiques effectives, et alerter l'opinion et les pouvoirs publics sur l'évolution restrictive dans l'application du droit au séjour pour raison médicale, les critères médicaux de demande retenus par le Comede étant stables sur la période.

La méthode utilisée consiste à caractériser chaque réponse préfectorale et juridictionnelle à chaque demande de carte de séjour, l'exhaustivité du recueil des réponses permettant d'éviter des biais de sélection. Chaque enregistrement effectué par le Comede correspond à un accord ou un refus documenté de la part de la préfecture et/ou de la juridiction compétente. Les variables renseignées concernent les caractéristiques du demandeur et de la demande effectuée. Les données recueillies sur la période 2002-2008 concernent 546 personnes suivies pour une ou plusieurs affections graves (713 au total, soit en moyenne 1,3 affection renseignée pour chaque demandeur), soit $2 \%$ des patients du Comede sur la période. Les démarches ont eu lieu dans quatorze départements, principalement en Île-deFrance. L'analyse statistique croisée des réponses avec les affections en cause conduit à la constitution d'un échantillon de 1230 enregistrements documentés ${ }^{(3)}$. 


\section{Droit au séjour des étrangers malades, évolution du taux d'accords}
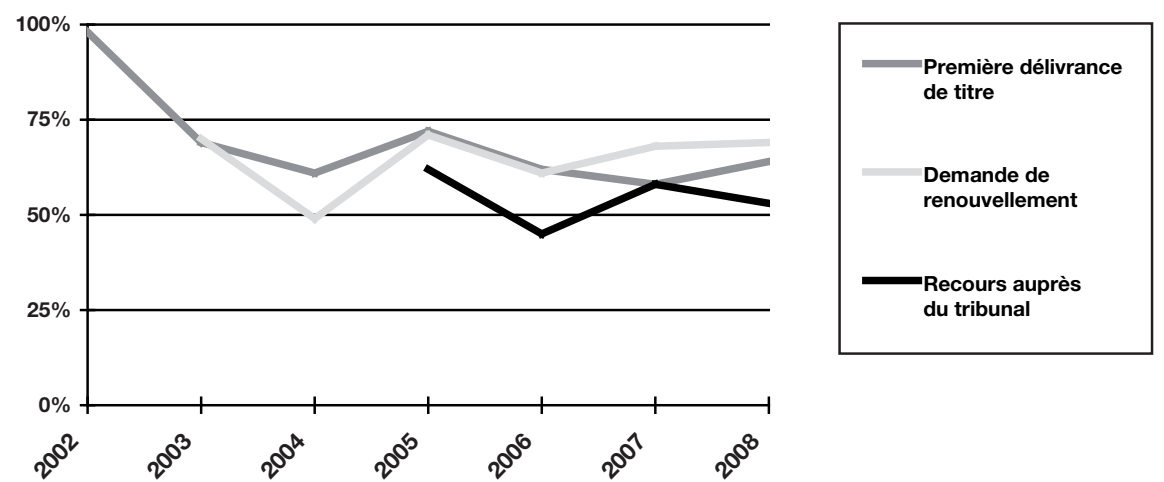

Proche de 100 \% jusqu'en 2002, les taux d'accords préfectoraux chutent en deux ans à $61 \%$ (en première délivrance) et à $49 \%$ (en renouvellement). Par la suite, les taux préfectoraux vont osciller autour de deux accords sur trois pour les préfectures, et de un sur deux pour les recours contentieux contre un refus préfectoral. Cette évolution a été observée en dehors de toute modification législative et réglementaire "officielle". Dans la mesure où, jusqu'en 2007, la quasi-totalité des décisions préfectorales respectaient les avis médicaux, ces résultats sont significatifs de l'impact des débats politiques et militants contradictoires sur l'élaboration des avis médicaux rendus par les MISP. Depuis 2004, la baisse des taux d'accords semble avoir été enrayée dans la plupart des départements, suite à la mobilisation des professionnels de santé et des associations, et à la publication en 2005 par le ministère de la Santé d'une circulaire rappelant la nécessité pour les MISP de protéger les étrangers vivant avec le VIH contre le renvoi vers un pays où l'accès aux soins ne peut être garanti.

\section{Médecins malgré tout}

Dans la préface d'un dossier de la revue Santé publique ${ }^{(4)}$, Moncef Marzouki s'interrogeait en 2001 sur le non-engagement des médecins pour le respect des droits humains en Tunisie. Médecin de santé publique ayant subi les persécutions du régime au pouvoir avant d'être exilé, il s'y étonnait du peu de mobilisation du milieu médical et de la primauté du paradigme biotechnique sur le paradigme 
social de la santé, avant de conclure : "En fait, nous autres médecins n'avons pas plus de raison de défendre les droits de la personne que les boulangers, les épiciers, les camionneurs ou les gardiens de prison. Nous n'avons pas moins de raisons non plus."

L'évolution du droit au séjour pour raison médicale tient pour beaucoup au positionnement des médecins à côté des acteurs plus traditionnels du droit des étrangers : administration, associations, juridictions et avocats. Et face aux restrictions observées depuis quelques années dans l'application de ce droit par l'administration, c'est bien souvent la capacité des médecins à appréhender les aspects déontologiques et éthiques de cette question complexe qui détermine le résultat des demandes. En outre, le médecin agissant à la demande d'un "patient" étranger déjà soutenu dans ses démarches par

\section{La présomption de fraude à l'égard des étrangers malades s'est étendue progressivement aux intervenants médicaux et associatifs qui leur viennent en aide.} une association ou un avocat, ces trois acteurs doivent apprendre à concerter leurs actions et à "travailler ensemble" au-delà de leurs différences de culture professionnelle.

Il n'est pas rare, en effet, de recueillir les plaintes d'un travailleur social, d'un avocat ou d'un professionnel associatif: "Le médecin de Monsieur X., il ne veut même pas faire de certificat médical pour son patient qui est pourtant très malade." Il n'est pas moins rare d'entendre des

médecins ironiser: "J'ai encore eu l'assistant socialll'avocat de Madame Z. au téléphone : il ne veut pas comprendre qu'elle est guérie/qu'elle n'aurait aucun problème pour se soigner dans son pays." Les intervenants, médecins et personnels des services sociaux et juridiques, des pôles ressources du Comede et de l'Espace Santé Droit partenariat du Comede et de la Cimade - diffusent quotidiennement des informations en matière de santé à des non-soignants et les textes réglementaires de référence à des médecins, et tentent autant que possible de susciter un dialogue pluridisciplinaire autour de la mise en ceuvre de la procédure de régularisation pour raison médicale.

Médecins praticiens (qui délivrent les rapports et certificats requis) comme Médecins inspecteurs de santé publique (c'est le MISP qui transmet, dans le respect du secret médical, son "avis" à la préfecture) se retrouvent aux prises avec une responsabilité qui déborde largement le champ de leur formation initiale: si l'évaluation du pronostic médical d'une affection relève de l'expertise technique d'un médecin, la traduction de ce pronostic en risque "d'exceptionnelle gravité" - qui n'a été précisée par aucun texte réglementaire - se situe sur le plan de l'éthique. De la même façon, en l'absence de données fiables et exhaustives sur l'accès effectif aux 
soins de santé dans la plupart des pays, l'évaluation du risque d'insuffisance des soins adéquats en cas de retour tient autant à ses connaissances en santé publique qu'à ses propres repères déontologiques.

Il est remarquable que ces médecins praticiens, et plus particulièrement les praticiens hospitaliers, les médecins agréés, les médecins des Unités de consultations et de soins ambulatoires (UCSA) des établissements pénitentiaires ou encore les médecins conventionnés pour intervenir dans les centres de rétentions administratives ne soient destinataires d'aucune information officielle spécifique sur les procédures de protection des étrangers malades vivant en France pour l'application desquelles ils sont pourtant expressément désignés par la réglementation : souvent cité en référence dans les circulaires du ministère de la Santé, le Guide Comede vient pallier là un déficit criant. À l'exception de quelques individus qui se sont documentés par leurs propres moyens, ces médecins ont été désignés comme éléments centraux d'un dispositif qu'on n'a pas pris le soin de leur expliciter. Ainsi, articles de codes, règlements et circulaires sont souvent diffusés par les associations.

Pour rompre cet isolement, des initiatives ont vu le jour dans tel réseau villehôpital ou tel service social hospitalier en partenariat avec des associations spécialisées... En 2005, les médecins intervenants en rétention administrative depuis 1999 , et donc en charge de la mise en ceuvre de la protection des étrangers malades contre la reconduite à la frontière, ont décidé de se rassembler au sein de la Fédération des unités médicales dans les centres de rétention administrative (Fumcra), et le thème du "droit des étrangers" occupe chaque année une place plus importante dans leurs rencontres.

Conséquence prévisible du caractère temporaire de la protection accordée (la carte de séjour doit être renouvelée chaque année), la montée en charge du dispositif a pourtant favorisé une suspicion croissante de la part des zélateurs de la "maitrise" de l'immigration. La présomption de fraude à l'égard des étrangers malades s'est étendue progressivement aux intervenants médicaux et associatifs qui leur viennent en aide: médecins traitants non hospitaliers (diffusion de listes de médecins "agréés" par la préfecture), associations de soutien aux étrangers (contrôle illégal de leur "agrément" en matière de domiciliation par les préfectures), médecins "agréés" (dont les "abus de délivrance de certificats médicaux" sont évoqués par une circulaire du ministère de l'Intérieur en janvier 2003) et, enfin, les MISP (soupçonnés de "complaisance" lors d'un débat à l'Assemblée nationale en juillet 2003). Après cinq premières années d'application de la loi favorable aux malades, le droit au séjour pour raison médicale devient la cible d'attaques répétées de la part des gouvernements successifs depuis 2003. 


\section{Du contrôle des malades à celui des médecins}

En mars 2007, contre l'avis des associations mais également du ministère de la Santé, le Comité interministériel de contrôle de l'immigration (CICI) décide la mise en ligne sur le site Intranet ministériel de "fiches-pays" indiquant l'existence de traitements et recommandant aux MISP, en contradiction avec la loi, de ne pas tenir compte de l'accessibilité effective aux soins. Elaborées par des médecins employés par les ministères de l'Immigration et des Affaires étrangères, ces fiches sont néanmoins utilisées par un certain nombre de MISP pour des décisions préfectorales de refus entraînant des recours de plus en plus nombreux. Dix ans après la création du droit au séjour des étrangers malades, et à l'instar des pratiques du droit d'asile, les juridictions de recours apparaissent plus favorables aux étrangers malades que les administrations en charge des procédures initiales.

L'analyse des données du Comede par département et par nationalité met en évidence des discriminations à la fois spatiales et nationales dans l'accès à la protection juridique pour les étrangers malades, ce qui renvoie à un phénomène bien connu en matière de droit des étrangers lors des périodes de crispation sur l'immigration : plus la demande est forte, plus l'accès aux droits se restreint. Les tentatives de suppression de ce droit par voie législative ou réglementaire ayant été abandonnées devant le risque politique consistant à "renvoyer des malades mourir dans leur pays ${ }^{(5)}$,", les autorités en charge du "contrôle de l'immigration" développent alors une stratégie de contournement du droit s'attachant tout particulièrement à discréditer non plus seulement les malades, mais désormais aussi les médecins.

Avec la "médicalisation" de l'asile, pour maîtriser l'immigration, il faut aussi pouvoir maîtriser les médecins qui soignent les étrangers. Déjà observé depuis 2003, la tentation du contrôle des avis médicaux par les autorités de police des étrangers s'amplifie en 2007 : de nombreuses préfectures refusent d'instruire la demande de carte de séjour lorsque le "rapport médical" n'émane pas d'un "médecin agréé ou un praticien hospitalier" ${ }^{\prime \prime()}$. Dans le même temps, plusieurs médecins voient leur agrément supprimé dans un contexte de suspicion croissante à l'égard des pratiques médicales ${ }^{(7)}$ et de pression des préfectures sur les MISP, qui en appellent fin 2007 au soutien de leur ministre de tutelle, celui de la Santé : "[Ces pressions] sinscrivent dans une démarche globale dintimidation retransmise par certains directeurs, eux-mêmes pressés par leurs préfets, qui ont été récemment convoqués pour des chiffres de reconduite à la frontière considérés comme insuffisants ${ }^{(8)}$."

C'est que, pour les plus hautes autorités de l'État, l'existence même d'un droit au séjour fondé sur des objectifs de protection de la santé reste constamment remise en 
cause. Début 2008, le rapport Mazeaud commandé par le ministre de l'Immigration propose à nouveau de supprimer le titre "étranger malade" : "Ces dispositions ont remplacé en quelque sorte, en pratique, les dispositifs propres à l'asile politique, à l'asile territorial ou au mariage avec un ressortissant français, dispositifs qui ont fait l'objet d'un dévoiement relatif, ce qui a conduit le législateur à intervenir à plusieurs reprises : en résumé, l'étranger est aujourd'hui malade et n'hésite pas à solliciter une carte de séjour à ce titre. Le nombre de dossiers contentieux en rapport avec l'application de ce texte est impressionnant. Beaucoup de dossiers ne valent d'ailleurs rien, masse qui doit sans doute refléter le nombre excessif de demandes présentées en préfecture à ce titre. On peut se demander s'il n'y aurait pas lieu de 'déconnecter' purement et simplement ce type de carte de séjour des titres de plein droit."

Fin 2008, dans la préface du dernier rapport du CICI au Parlement, le ministre de l'Immigration explique que la "nouvelle politique française de l'immigration repose sur trois principes fondateurs: souveraineté, justice et légalité”. Le principe de "légalité" s'applique à "l'étranger en situation irrégulière [qui] a vocation à être reconduit dans son pays d'origine, autant que possible de manière volontaire ou, s'il le faut, de manière contrainte". Rien n'est dit sur la protection que la loi garantit également à une partie de ces "étrangers en situation irrégulière" lorsqu'ils remplissent l'un des critères relatifs à la "vie privée et familiale" énoncés par l'article L. 313-11 du code de l'entrée et du séjour des étrangers et du droit d'asile.

Les baisses observées dans le champ du droit d'asile et du droit au séjour pour raison médicale marquent, selon le ministre, "le succès des réformes engagées par le gouvernement en 2003”, les titres de séjour destinés aux réfugiés et aux étrangers malades étant regroupés de manière significative dans une catégorie dénommée "humanitaire". 


\section{Sortir du silence}

Face aux difficultés qu'ils ont vite rencontrées dans l'application de la procédure "étrangers malades", les médecins ont tout d'abord répondu chacun de manière individuelle, s'appuyant notamment sur les règles de la déontologie médicale en faveur de la continuité des soins pour tous, mais aussi, pour les MISP, de l'indépendance de leurs décisions ${ }^{(9)}$. Mais les pressions croissantes et les mises en cause publiques survenues ces dernières années ont conduit un nombre croissant d'entre eux à répondre par la même voie, dénonçant publiquement le climat d'intimidation à leur égard et revendiquant leur rôle en matière de protection de la santé. Outre la lettre ouverte du syndicat des MISP, deux pétitions de médecins ont également été publiées au cours des derniers mois. L'une d'entre elle a fait suite à la suppression de l'agrément de deux psychiatres clermontois par le préfet du Puy-de-Dôme ; l'autre a été rendue publique en juin 2008 dans le cadre d'une conférence de presse organisée conjointement avec des associations de malades et les associations de l'ODSE.

À l'occasion des dix ans de la reconnaissance du droit au séjour des étrangers malades, cinq cents médecins, hospitaliers ou libéraux, agréés ou non, dans une pétition intitulée "La déontologie médicale est universelle : elle s'applique aussi pour les malades étrangers", déclaraient : "Il est temps que les autorités préfectorales cessent de stigmatiser l'irresponsabilité' des médecins intervenant auprès des malades étrangers. Non, nous ne sommes pas 'complaisants' ou 'angéliques' lorsque nous signalons la gravité de la maladie de nos patients à nos confrères MISP. Non, nous ne sommes pas 'militants' ou 'engagés' lorsque nous estimons un pronostic vital dans une perspective d'absence de soins appropriés, nous faisons notre devoir de médecins [...]. Nous ne pouvons accepter que, au mépris de la loi, les principes de la déontologie médicale s'effacent devant les mécanismes de la 'lutte contre l'immigration'."

\section{Conclusion}

Nos organisations sont convaincues que les médecins ont à transmettre une culture de santé aux travailleurs sociaux et associatifs en charge de l'accompagnement des migrants dans l'accès à leurs droits et que, réciproquement, les mêmes médecins peuvent plus sereinement recourir aux principes de la déontologie médicale si on leur explique les imbroglios d'une demande de titre de séjour auprès d'une préfecture ou les conséquences d'une décision de refus et/ou d'une reconduite à la frontière. Elles sont fermement opposées aux politiques publiques à l'ceuvre qui renvoient dos à dos logique de sécurité et logique de santé et diffusent de fausses informations sur l'accessibilité effective des traitements dans les pays d'origine : ces politiques interdi- 
sent ainsi la réflexion pluridisciplinaire indispensable à la détermination du besoin ou non de maintien en France d'un étranger malade pour la continuité de ses soins. C'est l'irresponsabilité de l'ensemble des acteurs qui est organisée.

Les considérations d'ordre médical, si elles ne sont pas exclusives, sont centrales dans le dispositif de droit au séjour des étrangers malades. Les soutiens des étrangers, les soignants et les personnels des services sociaux et juridiques tentent de s'organiser, seuls, pour appréhender l'ensemble des enjeux en intelligence et de manière complémentaire. Il est désormais urgent que les plus hautes autorités de l'État procèdent au même rapprochement. La politique de délégitimation du droit à la santé des étrangers vivant en France, orchestrée par le ministère de l'Immigration, ne saurait rester sans contrepoids indéfiniment. Les recommandations claires du ministère de la Santé concernant les étrangers touchés par le VIHsida ne peuvent rester une exception : ce ministère a une parole forte à faire entendre sur les enjeux de santé, tant publique qu'individuelle, des populations migrantes vivant en France. Il n'est jamais trop tard.

\section{Notes}

1. Article 12 bis $11^{\circ}$ de l'ordonnance du 2 novembre 1945 devenu l'article L313-11 $11^{\circ}$ du code de l'entrée, du séjour des étrangers et du droit d'asile créé en 2004.

2. A. Veïsse, "Le médecin, la santé et le séjour des étrangers", Plein droit, 69, 2006.

3. "Étrangers malades : menaces sur le droit au séjour", Maux d'exil, n²6, mars 2009.

4. M. Marzouki "Le non-engagement scientifique pour le respect des droits fondamentaux de la personne", Santé publique, vol. 13, 2001.

5. Pétition lancée en avril 2007 par l'ODSE et dix personnalités du domaine de l'éthique et de la santé publique.

6. Ces refus s'appuient sur l'exigence d'appliquer de manière nouvellement restrictive un arrêté du 8 juillet 1999 relatif aux conditions d'établissement des avis médicaux concernant les étrangers malades.

7. En 2007 , trois médecins psychiatres soignant des étrangers malades dans le Puy-de-Dôme se sont vu supprimer leur agrément par le préfet, au motif notamment que l'un d'entre eux "a été amené à produire des rapports médicaux concernant des étrangers comme praticien agréé, alors que ces étrangers étaient également ses patients dans le cadre de son exercice privé". Dans le Bas-Rhin, un autre médecin psychiatre s'est vu révoquer par le préfet pour avoir transmis "trop" de rapports médicaux relatifs à la continuité des soins de ses patients étrangers.

8. Syndicat des médecins inspecteurs de santé publique, communiqué du 8 octobre 2007, www.smisp.fr

9. Article 47 du code de déontologie médicale (R4127-47) et 95 du code de la santé publique (R4127-95). 